\title{
On approximate closed form solutions of linear ordinary differential equations
}

\author{
F. Viera* \\ (Received 21 October 2005; revised 14 August 2006)
}

\begin{abstract}
Approximate closed form solutions of a linear ordinary differential equation are obtained using piecewise approximations of the arbitrary coefficient function. We show how to obtain expressions for the general solution and the eigenvalue equation. An example is given with specific boundary conditions typical of a range of problems in mathematical physics and engineering. The method is robust and accurate and can be used as a complement to standard numerical techniques. The function representing the approximate solution has a simple form and can be used like a standard function in calculations that require the solution of the differential equation.
\end{abstract}

${ }^{*}$ School of Science \& Technology, Charles Sturt University, Wagga Wagga, Australia. mailto:fviera@csu.edu.au

See http://anziamj.austms.org.au/V47EMAC2005/Viera for this article, (c) Austral. Mathematical Soc. 2006. Published September 18, 2006. ISSN 1446-8735 


\section{Contents}

1 Introduction

C246

2 Problem formulation

C247

3 The general solution

C249

4 Infinite domain example

C252

5 Conclusions

C259

References

C260

\section{Introduction}

There are situations when it is desirable to have closed form solutions of an ordinary differential equation (ODE) even if such solutions are only approximate. Eigenvalue problems are a typical example. In this case the ODE depends on an eigenvalue $\lambda$ and the solution may only exist for specific values of $\lambda$. It is desirable to have an analytic expression from which to calculate the eigenvalues.

There are other cases when the solution is required in subsequent calculations. The ODE may be the result of the application of Fourier or Laplace transforms to a linear partial differential equation. In this case the coefficients will be functions of the transform variable and it is convenient to have a closed form expression in order to attempt an inversion of the transform.

In this article we study the linear differential equation

$$
\frac{d^{2} \psi}{d x^{2}}+f(x ; \lambda) \psi=0,
$$


and show how to obtain approximate closed form solutions by approximating $f(x ; \lambda)$ by a piecewise function. We introduce a partition of the domain $D$ where the equation is defined and choose local approximating functions so the resulting ODEs have closed form solutions.

As early as 1912, Rayleigh [1] used a similar approach in the solution of the wave equation by the layer method. Chernyak [2] used the method to solve a problem concerning the scattering of a particle by two close potentials in quantum mechanics and more recently, Kalotas and Lee [3] used piecewise constant coefficients to study the scattering of a particle by an arbitrary onedimensional potential barrier. With the advent of computer algebra systems it is now feasible to extend the procedure in a systematic way to obtain approximate closed form solutions of a number of linear problems.

In Section 2 we formulate the problem in terms of a general function $f(x ; \lambda)$ and identify the form of the approximate solution. In Section 3 we calculate the general solution in terms of two arbitrary constants and express it in a simple form that can be used like a standard function.

In Section 4 we give an example that illustrates the potential applications of the method. The problem deals with the time independent Schrödinger's equation in an infinite domain. Finally, in Section 5 we summarize the main conclusions, identify possible extensions and discuss a few issues that are important for the successful computer implementation of the method.

\section{Problem formulation}

We wish to find approximate solutions of equation (1) in a domain $D$ which we assume to be infinite. We divide the problem formulation into four steps:

1. Introduce a partition of $D$ consisting of $N+1$ points $x_{1}, x_{2}, \ldots, x_{N+1}$ 
and consider the $N+2$ disjoint intervals

$$
\begin{aligned}
I_{n} & =\left[x_{n}, x_{n+1}\right], \quad n=1,2, \ldots, N, \\
I_{0} & =\left(-\infty, x_{1}\right], \quad I_{N+1}=\left[x_{N+1}, \infty\right) .
\end{aligned}
$$

2. Select a sequence of continuous functions $\left\{f_{n}(x ; \lambda)\right\}, n=0,1, \ldots, N+$ 1 , that are local approximations to the coefficient function $f(x ; \lambda)$ on the interval $I_{n}$. Construct the restriction $\hat{f}_{n}$ of $f_{n}$ :

$$
\hat{f}_{n}(x ; \lambda)=f_{n}(x ; \lambda) \chi_{n}, \quad n=0,1, \ldots, N+1,
$$

where

$$
\chi_{n}= \begin{cases}1, & \text { if } x \in I_{n}, \\ 0, & \text { otherwise }\end{cases}
$$

is the characteristic function of the interval $I_{n}$. Then the function

$$
f_{N}(x ; \lambda)=\sum_{n=0}^{N+1} \hat{f}_{n}(x ; \lambda),
$$

is a piecewise approximation of $f(x ; \lambda)$ in the domain $D$. In general, $f_{N}(x ; \lambda)$ will be a piecewise continuous function with possibly a finite number of finite discontinuities at the partition points $\left\{x_{n}\right\}$. In the example in Section 4 we use interpolating linear functions through the points $\left[x_{n}, f\left(x_{n} ; \lambda\right)\right]$ and therefore $f_{N}$ will be continuous with a piecewise continuous derivative on $D$.

3. Construct the sequence of local differential operators

$$
\hat{L}_{n} \equiv \frac{d^{2}}{d x^{2}}+\hat{f}_{n}(x ; \lambda), \quad n=0,1, \ldots, N+1 .
$$

For each $n$ the local operators have an associated local equation

$$
\hat{L}_{n} \hat{\psi}_{n}=0, \quad n=0,1, \ldots, N+1,
$$

with solution $\hat{\psi}_{n}(x ; \lambda)=\psi_{n}(x ; \lambda) \chi_{n}$ valid on $I_{n}$. I emphasize that the sequence $\left\{f_{n}(x ; \lambda)\right\}$ must be chosen so that the local equation (4) has known closed form solutions. 
4. Finally, let the global function consist of the sum of the local solutions

$$
\psi_{N}(x ; \lambda)=\sum_{n=0}^{N+1} \hat{\psi}_{n}(x ; \lambda)
$$

on the original domain $D$. It is now easy to prove the following theorem.

Theorem 1 The global function $\psi_{N}(x ; \lambda)$ is an exact solution of the equation $L_{N} \psi_{N}=0$ where the global operator

$$
L_{N} \equiv \frac{d^{2}}{d x^{2}}+f_{N}(x ; \lambda) \text { on } D,
$$

and $f_{N}(x ; \lambda)$ is given in $(3)$.

The proof, which is omitted, uses the linearity of $L_{N}$ and the orthogonality of $\chi_{n}$ with respect to real multiplication on the given domain $D$. This result gives a formula to construct the approximate solution $\psi_{N}$ once $f(x ; \lambda)$ is approximated by $f_{N}(x ; \lambda)$.

\section{The general solution}

According to Theorem 1, to find the approximate solution of (1) we must consider the general solution of the local equation (4) valid on $I_{j}$ :

$$
\hat{\psi}_{j}(x ; \lambda)=\left[a_{j} \alpha_{j}(x ; \lambda)+b_{j} \beta_{j}(x ; \lambda)\right] \chi_{j}, \quad j=0,1, \ldots, N+1,
$$

where $\alpha_{j}(x ; \lambda)$ and $\beta_{j}(x ; \lambda)$ are two linearly independent solutions, and where $a_{j}$ and $b_{j}$ are two arbitrary constants.

We require continuity of $\psi$ and its derivative at each partition point $x=$ $x_{j}$, that is

$$
\hat{\psi}_{j-1}\left(x_{j} ; \lambda\right)=\hat{\psi}_{j}\left(x_{j} ; \lambda\right)
$$




$$
\hat{\psi}_{j-1}^{\prime}\left(x_{j} ; \lambda\right)=\hat{\psi}_{j}^{\prime}\left(x_{j} ; \lambda\right), \quad j=1,2, \ldots, N+1,
$$

where the prime indicates differentiation with respect to $x$. Substituting (6) into (7) and solving for $a_{j-1}$ and $b_{j-1}$ in terms of $a_{j}$ and $b_{j}$ we get

$$
\mathbf{a}_{j-1}=M_{j} \mathbf{a}_{j}, \quad j=1,2, \ldots, N+1 .
$$

The vector $\mathbf{a}_{j}$ and the matrix $M_{j}$ are

$$
\mathbf{a}_{j}=\left[\begin{array}{l}
a_{j} \\
b_{j}
\end{array}\right], \quad M_{j}=\left[\begin{array}{ll}
M_{11}^{(j)} & M_{12}^{(j)} \\
M_{21}^{(j)} & M_{22}^{(j)}
\end{array}\right]
$$

where

$$
\begin{array}{ll}
M_{11}^{(j)}=\frac{1}{\Delta_{j}} W\left(\alpha_{j}, \beta_{j-1}\right), & M_{12}^{(j)}=\frac{1}{\Delta_{j}} W\left(\beta_{j}, \beta_{j-1}\right), \\
M_{21}^{(j)}=\frac{1}{\Delta_{j}} W\left(\alpha_{j-1}, \alpha_{j}\right), & M_{22}^{(j)}=\frac{1}{\Delta_{j}} W\left(\alpha_{j-1}, \beta_{j}\right), \\
\Delta_{j}=W\left(\alpha_{j-1}, \beta_{j-1}\right), &
\end{array}
$$

and where $W(f, g)=f g^{\prime}-f^{\prime} g$ is the Wronskian of $f$ and $g$. Note that $\Delta_{j} \neq 0$ since $\alpha_{j-1}$ and $\beta_{j-1}$ are two linearly independent functions. All of the above expressions are evaluated at the partition points $x=x_{j}$ and are valid for $j=1,2, \ldots, N+1$.

Expanding the system (8) it may be shown that the coefficient vectors $\mathbf{a}_{j}$ may be expressed in terms of the vector $\mathbf{a}_{N+1}$ :

$$
\mathbf{a}_{j}=P_{j+1} \mathbf{a}_{N+1}, \quad P_{j+1}=\prod_{k=j+1}^{N+1} M_{k}, \quad j=0,1, \ldots, N .
$$

Then the matrix $P_{j}$ satisfies the recurrence relation

$$
P_{j-1}=M_{j-1} P_{j}, \quad j=2,3, \ldots, N+1,
$$




$$
P_{N+1}=M_{N+1} .
$$

This recurrence relation is very important for the actual implementation of the method and allows the efficient computation of all the coefficients in (10). Substituting $\mathbf{a}_{j}$ into (6) and then substituting the result into (5) gives the approximate general solution

$$
\psi_{N}(x ; \lambda)=\sum_{j=0}^{N+1}\left[a_{j} \alpha_{j}(x ; \lambda)+b_{j} \beta_{j}(x ; \lambda)\right] \chi_{j},
$$

in terms of the arbitrary vector

$$
\mathbf{a}_{N+1}=\left[\begin{array}{l}
a_{N+1} \\
b_{N+1}
\end{array}\right] .
$$

Remark I emphasize that the summation in (12) is a disjoint sum and that once the value of $x$ is specified we only have to identify the corresponding interval $I_{j}$ where $x$ belongs and compute only that term in the sum to get the value of the solution at that point. All other terms will be zero by definition of $\chi_{j}$.

This means that we can rewrite (12) using a single term as described in the following proposition.

Proposition 2 If the solutions $\alpha_{j}(x ; \lambda)$ and $\beta_{j}(x ; \lambda)$ of the local equation (4) are obtained from partitioning single functions $A(x ; \lambda)$ and $B(x ; \lambda)$, respectively, then the solution (12) can be written in the simpler form

$$
\psi_{N}(x ; \lambda)=\hat{a} A(x ; \lambda)+\hat{b} B(x ; \lambda),
$$

where

$$
(\hat{a}, \hat{b})=\sum_{j=0}^{N+1}\left(a_{j}, b_{j}\right) \chi_{j}
$$

are piecewise constant functions of $x$. 
We see an example of how this is done in equation (21) in the next section.

\section{Infinite domain example}

We now consider the coefficient function

$$
f(x ; \lambda)=\lambda+U_{0} \operatorname{sech}^{2} x .
$$

In this case equation (1) becomes the time independent Schrödinger's equation with potential $u(x)=-U_{0} \operatorname{sech}^{2} x$ where $U_{0}$ is a constant. The boundary conditions to be satisfied are that the solution is finite as $x \rightarrow \pm \infty$. This is the classic quantum mechanics problem of a particle bound by the potential $u(x)$.

This equation has an exact solution (see Landau and Lifshitz [4] for example) that will be used to check the calculations and compare with the approximate expression. Because the domain $D$ is infinite there are only a finite number $K$ of eigenvalues, $K=\left[\left(U_{0}+1 / 4\right)^{1 / 2}-1 / 2\right]+1$, where the square bracket $[\ldots]$ indicates the integer part and the +1 is omitted when $\left(U_{0}+\frac{1}{4}\right)^{1 / 2}-\frac{1}{2}$ is an integer. Letting $\lambda=-\nu^{2}$, the exact eigenvalues are

$$
\nu_{k}=\left(U_{0}+1 / 4\right)^{1 / 2}-(k+1 / 2), \quad k=0,1, \ldots, K-1 .
$$

When $U_{0}=6$, Equation (16) predicts the two exact eigenvalues $\nu_{0}=2$, $\nu_{1}=1$. The corresponding exact eigenfunctions are

$$
\begin{aligned}
& \phi_{0}=c_{0} \operatorname{sech}^{2} x, \\
& \phi_{1}=c_{1} \tanh x \operatorname{sech} x,
\end{aligned}
$$

where $c_{0}$ and $c_{1}$ are normalization constants.

In order to calculate the approximate solution we use approximating functions (2) given by straight line segments,

$$
f_{n}(x ; \lambda)=m_{n}(\lambda) x+c_{n}(\lambda),
$$


TABLE 1: Two exact eigenvalues and the two corresponding approximate eigenvalues calculated numerically from equation (20) for $U_{0}=6$ and $N+1=$ 7 partition points. The average relative error is $2.3 \%$.

\begin{tabular}{cccc}
\hline$k$ & $\nu_{k}$ Exact & $\hat{\nu}_{k}$ Approximate & Relative error \\
\hline 0 & $\nu_{0}=2$ & $\hat{\nu}_{0}=1.926317$ & $3.8 \%$ \\
1 & $\nu_{1}=1$ & $\hat{\nu}_{1}=1.007832$ & $0.8 \%$ \\
\hline
\end{tabular}

joining the points $\left[x_{n}, f\left(x_{n} ; \lambda\right)\right]$ for $n=1,2, \ldots, N+1$. Then if $m_{n} \neq 0$ the local equations (4) have solutions in terms of Airy functions (Abramowitz and Stegun [5]) of the form

$$
\begin{aligned}
& \psi_{n}(x ; \lambda)=a_{n} \operatorname{Ai}\left(\xi_{n}\right)+b_{n} \operatorname{Bi}\left(\xi_{n}\right), \\
& \xi_{n}(\lambda)=-m_{n}(\lambda)^{-2 / 3}\left[m_{n}(\lambda) x+c_{n}(\lambda)+\lambda\right] .
\end{aligned}
$$

If $m_{n}=0$ the solutions are given by exponential or trigonometric functions. For example, in the semi-infinite intervals $I_{0}$ and $I_{N+1}$ the potential function $u(x)$ in (15) decays sufficiently fast at infinity so that

$$
f(x ; \lambda) \sim \lambda \text { as } x \rightarrow \pm \infty .
$$

In this case, the local eigenfunctions on $I_{0}$ and $I_{N+1}$ are

$$
\begin{aligned}
\psi_{0}(x ; \lambda) & =a_{0} \exp (-\nu x)+b_{0} \exp (\nu x), \\
\psi_{N+1}(x ; \lambda) & =a_{N+1} \exp (-\nu x)+b_{N+1} \exp (\nu x),
\end{aligned}
$$

where $\lambda=-\nu^{2}<0$. For a finite solution as $x \rightarrow \pm \infty$ we require both $a_{0}$ and $b_{N+1}$ to be zero. We set $b_{N+1}=0$ because, from (13), it is one of the arbitrary constants. However, in order to make $a_{0}=0$ as well, we have to consider the relationship between the coefficient vectors $\mathbf{a}_{0}$ and $\mathbf{a}_{N+1}$ which is given by (10) with $j=0$, namely $\mathbf{a}_{0}=P_{1} \mathbf{a}_{N+1}$, or in component form,

$$
\left[\begin{array}{l}
a_{0} \\
b_{0}
\end{array}\right]=\left[\begin{array}{ll}
P_{11}^{(1)} & P_{12}^{(1)} \\
P_{21}^{(1)} & P_{22}^{(1)}
\end{array}\right]\left[\begin{array}{l}
a_{N+1} \\
b_{N+1}
\end{array}\right] \text {. }
$$


Substituting $b_{N+1}=0$ into (19) gives $a_{0}=P_{11}^{(1)} a_{N+1}$ and $b_{0}=P_{21}^{(1)} a_{N+1}$. Therefore, for non-trivial solutions we require

$$
P_{11}^{(1)}(\lambda)=0 \text {. }
$$

This is the eigenvalue equation whose solution gives the discrete set of eigenvalues $\lambda_{n}, n=1,2, \ldots, K$. I point out that because the local solutions (6) depend on $\lambda$, the matrices $M_{j}$ and $P_{j}$ will also depend on $\lambda$. This means that the eigenvalue equation can be quite complicated and in most cases can only be solved numerically.

Figure 1 shows plots of the left hand side of (20) as a function of $\nu$ for three values of the potential amplitude, $U_{0}=1,4,6$, using $N+1=7$ partition points. The solid curve corresponds to $U_{0}=6$ and shows three intersections with the $\nu$-axis at $\hat{\nu}_{0} \approx 1.926, \hat{\nu}_{1} \approx 1.008$ and $\hat{\nu}_{2} \approx 0.209$. The first two are shown in Table 1 together with the two exact solutions predicted by (16).

Our approximate solution also has a third eigenvalue $\hat{\nu}_{2} \approx 0.209$. Equation (16), with $\nu_{k}=0.2$ and $U_{0}=6$, gives $k \approx 2$ which corresponds to the third eigenfunction. This is not surprising since we are only using an approximation to the coefficient function (15). However, we found that as $N$ increases, and therefore $f_{N}(x ; \lambda) \rightarrow f(x ; \lambda)$, the third eigenvalue $\hat{\nu}_{2} \rightarrow 0$ as expected. When $\nu=1$ the exact eigenvalue equation (16) can be solved for $U_{0}$ to give $U_{0}=(k+3 / 2)^{2}-1 / 4$. For $k=0,1,2,3, \ldots$ this equation gives values of $U_{0}=2,6,12,20, \ldots$. This behaviour is also exhibited by the approximate solution and can be observed in Figure 2 which plots the eigenvalue equation as a function of the potential amplitude $U_{0}$ for $\nu=1,2,3$. The solid curve, which represents the eigenvalue $\nu=1$, intersects the horizontal axis at values of $U_{0} \approx 2,6,11,18, \ldots$, in close agreement with the exact solution. Also note that for $\nu=2, U_{0} \approx 6$, as in Table 1 .

The approximate closed form eigenfunction (14) corresponding to $\lambda \equiv$ $\lambda_{1}=-\hat{\nu}_{1}^{2}$ can be written as

$$
\psi_{N}\left(x ; \lambda_{1}\right)=\hat{a} \operatorname{Ai}(\hat{\sigma} x+\hat{\tau})+\hat{b} \operatorname{Bi}(\hat{\sigma} x+\hat{\tau})+\hat{c} \exp (\hat{\rho} x),
$$




\section{Eigenvalue Equation}

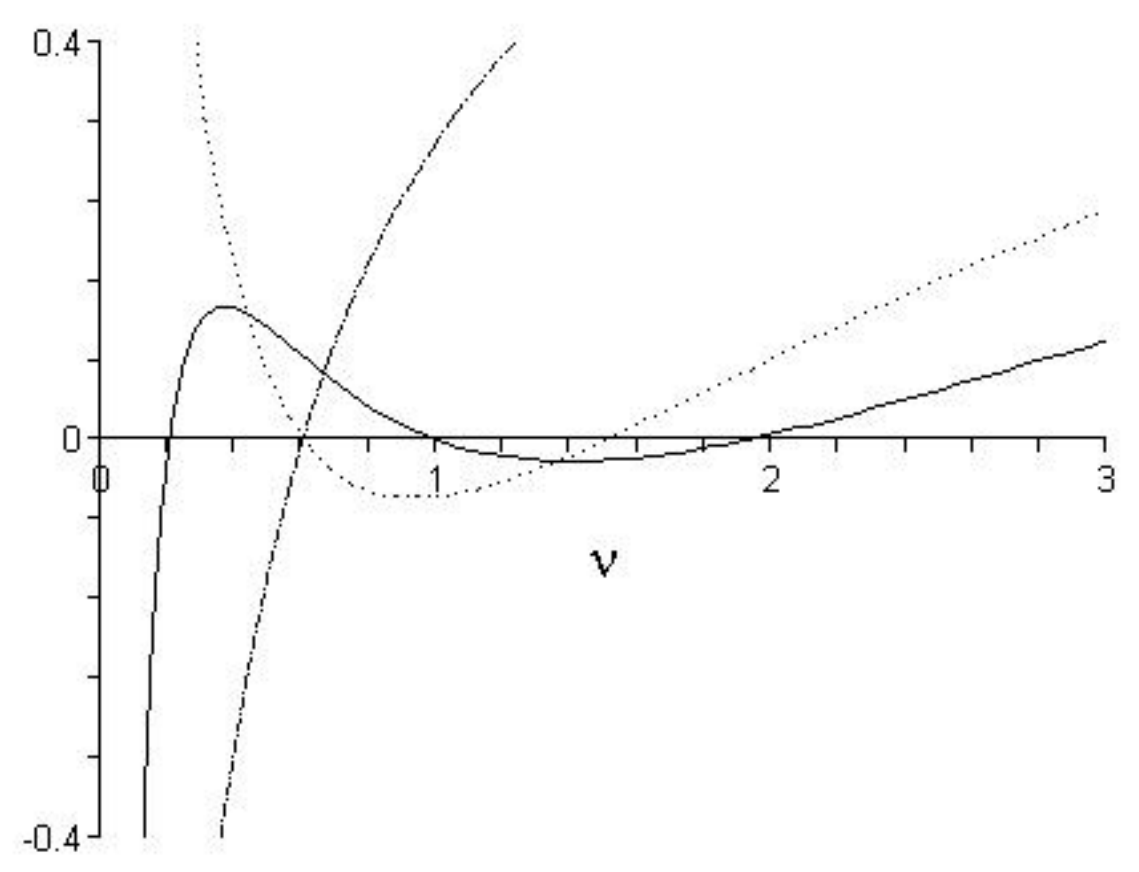

Figure 1: Left-hand Side of the eigenvalue equation (20) as a function of $\nu$ for three values of the potential amplitude: $U_{0}=6$ (solid), $U_{0}=4$ (dashed), $U_{0}=1$ (dotted). 


\section{Eigenvalue Equation}



Figure 2: Left-hand Side of the eigenvalue equation (20) as a function of the potential amplitude $U_{0}$ for three values of $\nu: \nu=1$ (solid), $\nu=2$ (dashed), $\nu=3$ (dotted). Note the intersections of the solid curve with the $U_{0}$-axis at $U_{0} \approx 2,6,11,18$. 
where $\operatorname{Ai}(z)$ and $\operatorname{Bi}(z)$ are the Airy functions introduced in equation (18),

$$
\begin{aligned}
& (\hat{a}, \hat{b}, \hat{\sigma}, \hat{\tau})=\sum_{j=1}^{6}\left(a_{j}, b_{j}, \sigma_{j}, \tau_{j}\right) \chi_{j}, \\
& \hat{c}=b_{0} \chi_{0}+b_{7} \chi_{7}, \quad \hat{\rho}=\sigma_{0} \chi_{0}+\sigma_{7} \chi_{7},
\end{aligned}
$$

and where

$$
\begin{array}{ll}
a_{0}=0, & b_{0}=-1, \\
a_{1}=-a_{6}=-0.438355 \times 10^{3}, & b_{1}=-b_{6}=-0.778714 \times 10^{-7}, \\
a_{2}=-a_{5}=-0.397119, & b_{2}=-b_{5}=-0.539939 \times 10^{-2}, \\
a_{3}=-a_{4}=-0.360504, & b_{3}=-b_{4}=-0.760902 \times 10^{-1}, \\
a_{7}=1, & b_{7}=0, \\
\sigma_{0}=-\sigma_{7}=1.007832, & \\
\sigma_{1}=-\sigma_{6}=-0.430951, & \tau_{1}=\tau_{6}=3.702027, \\
\sigma_{2}=-\sigma_{5}=-1.002459, & \tau_{2}=\tau_{5}=-1.776677, \\
\sigma_{3}=-\sigma_{4}=-1.504657, & \tau_{3}=\tau_{4}=-2.201542, \\
x_{1}=-x_{7}=-4, & \\
x_{2}=-x_{6}=-8 / 3, & \\
x_{3}=-x_{5}=-4 / 3, & \\
x_{4}=0, &
\end{array}
$$

where we recall that $N+1=7$ partition points and that $a_{0}=b_{7}=0$ from the boundary conditions. Figure 3 shows plots of $\psi_{N}\left(x ; \lambda_{1}\right)$ and the exact eigenfunction $\phi_{1}(x)$ in (17) as a function of $x$. The approximate solution is calculated at $\nu=\hat{\nu}_{1}$ from Table 1. Both functions are normalized to unity at their maximum values.

We found that the $L_{2}$ norm of the difference between the two functions was of the order of 0.1 in all calculations using $N+1=7$ partition points. The norm was also found to decrease as the number of partition points increased indicating that $\left\|\psi_{N}-\phi_{1}\right\| \rightarrow 0$ as $N \rightarrow \infty$. 


\section{Exact and Approximate Eigenfunctions}

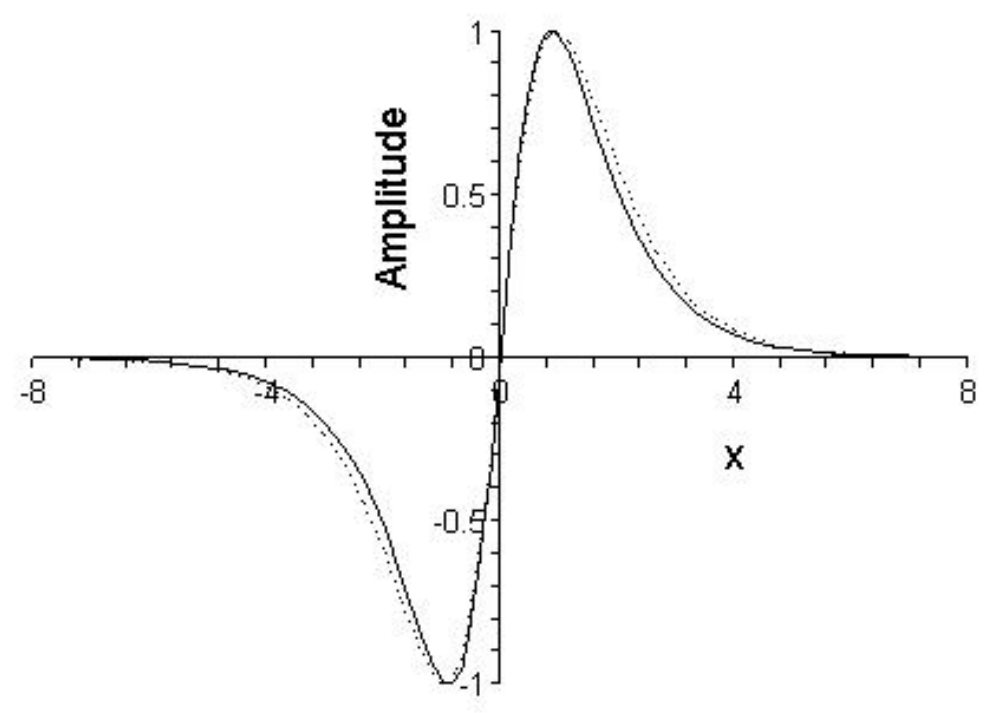

FIGURE 3: the exact (solid) and approximate (dashed) eigenfunctions corresponding to $\nu=\hat{\nu}_{1} \approx \nu_{1}$ as a function of $x$ for $U_{0}=6$ calculated from equations (17) and (21), respectively. The eigenfunctions are normalized to unity at their maximum values. 


\section{Conclusions}

We have shown that using a piecewise approximation of the function $f(x ; \lambda)$ it is possible to obtain approximate closed form solutions of the differential equation

$$
\frac{d^{2} \psi}{d x^{2}}+f(x ; \lambda) \psi=0 .
$$

We looked at an example in an infinite domain that has an exact solution and was compared with the approximate expression. We then showed how to obtain an analytic expression for the eigenvalue equation. However, the eigenvalue equation has a complicated dependence on $\lambda$ and in general it has to be solved numerically to obtain the eigenvalues.

The method can be extended in several ways. Firstly, it can be applied to two point boundary value problems in a finite domain $[a, b]$. Similar expressions to (20) can be obtained for the eigenvalue equation in this case. Secondly, the matrix formulation of Section 3 can be extended to include non-homogeneous equations with more than one coefficient function such as

$$
\frac{d^{2} \psi}{d x^{2}}+f(x) \frac{d \psi}{d x}+g(x) \psi=h(x) .
$$

Thirdly, other sequences of functions $\left\{\hat{f}_{n}\right\}$ given in (2) may be used to increase the accuracy of the method for a given partition size. The main criterion is that the local equations (4) must have known closed form solutions. For example, if $\left\{\hat{f}_{n}\right\}$ are quadratic functions then the approximate solution can be expressed in terms of hypergeometric functions.

Finally, the successful software implementation of the method using a computer algebra system requires that we use a top-down approach whenever possible. For example, calculation of the matrix products in (10) should be carried out with dummy symbols for the eigenfunctions. The actual expressions such as the Airy functions, should be substituted at the end after all matrix multiplications have been carried out. 
Acknowledgements: I thank the referees for their invaluable comments.

\section{References}

[1] Rayleigh Lord (Strutt J. W.), 1912, Proc. Roy. Soc. A, 86, 207. http: //www. journals.royalsoc.ac.uk/link. asp?id=eg142ju756873w13 $\mathrm{C} 247$

[2] Y. B. Chernyak, On a fitting technique approach in potential scattering theory, J. Phys. A: Math. Gen., Vol. 10, No 9, 1477, 1977. http://dx.doi.org/10.1088/0305-4470/10/9/009 C247

[3] T. M. Kalotas and A. R. Lee, A new approach to one-dimensional scattering, Am. J. Phys., Vol. 59, 48-52, 1991. http://dx.doi.org/10.1119/1.16705 C247

[4] L. D. Landau, E. M. Lifshitz, Quantum Mechanics. Non-Relativistic theory. 3rd Edn. London: Pergamon. 1977. C252

[5] M. Abramowitz and I. A. Stegun, Handbook of Mathematical Functions, Dover, New York, 1965. C253 